\title{
TOPOLOGICAL ENTROPY AND BLOCKING COST FOR GEODESICS IN RIEMANNIAN MANIFOLDS
}

\author{
EUGENE GUTKIN
}

\begin{abstract}
For a pair of points $x, y$ in a compact, riemannian manifold $M$ let $n_{t}(x, y)$ (resp. $s_{t}(x, y)$ ) be the number of geodesic segments with length $\leq t$ joining these points (resp. the minimal number of point obstacles needed to block them). We study relationships between the growth rates of $n_{t}(x, y)$ and $s_{t}(x, y)$ as $t \rightarrow \infty$. We derive lower bounds on $s_{t}(x, y)$ in terms of the topological entropy $h(M)$ and its fundamental group. This strengthens the results of Burns-Gutkin [2] and Lafont-Schmidt [13. For instance, by [2, 13, $h(M)>0$ implies that $s$ is unbounded; we show that $s$ grows exponentially, with the rate at least $h(M) / 2$.
\end{abstract}

\section{INTRODUCTION}

By a riemannian manifold we will always mean a closed, complete, connected, infinitely differentiable, riemannian manifold. Let $M$ be a riemannian manifold. By a geodesic $\gamma \subset M$ we will mean an oriented geodesic segment; thus, $\gamma$ has endpoints $x, y \in M$ and a positive length, $|\gamma|$. (We allow $x=y$.) If $z \in M$ is an interior point of $\gamma$, we say that $\gamma$ passes through $z$. For $x, y \in M$ and $0<t$ we denote by $G_{t}(x, y)$ the set of geodesics with endpoints $x, y$ and length at most $t$. Let $\Gamma_{t}(x, y) \subset$ $G_{t}(x, y)$ be the subset of those $\gamma \in G_{t}(x, y)$ that do not pass through either $x$ or $y$. We set $G(x, y)=\cup_{t \in \mathbb{R}_{+}} G_{t}(x, y), \Gamma(x, y)=\cup_{t \in \mathbb{R}_{+}} \Gamma_{t}(x, y)$. We will say that the geodesics in $G(x, y)$ (resp. $\Gamma(x, y)$ ) join (resp. connect) $x$ with $y$.

A finite set $B \subset M \backslash\{x, y\}$ is a blocking set for $\Gamma_{t}(x, y)$ if every $\gamma \in \Gamma_{t}(x, y)$ passes through a point in $B$. Let $s_{t}(x, y) \leq \infty$ be the minimal cardinality of a blocking set, and set $s(t)=\sup _{x, y \in M} s_{t}(x, y)$. We say that $t \mapsto s_{t}(x, y)$ is the blocking threshold function for $x, y \in M$, and that $t \mapsto s(t)$ is the blocking cost function for $M$.

Date: November 2, 2018.

1991 Mathematics Subject Classification. 37D40, 53C22.

Key words and phrases. riemannian manifold, connecting geodesics, blocking threshold, counting of geodesics, topological entropy. 
The framework of security for riemannian manifolds concerns the question of blocking all of geodesics in $\Gamma(x, y)$ by a finite set [2, 11, 12, 13. A pair $x, y \in M$ is secure if there is a finite blocking set for $\Gamma(x, y)$; otherwise it is insecure. A manifold $M$ is secure if every pair of points in $M$ is secure. Otherwise $M$ is insecure. If $M$ is secure, and there is a uniform upper bound on the cardinality of minimal blocking sets, then $M$ is uniformly secure. On the other hand, $M$ is totally insecure if all pairs $x, y \in M$ are insecure.

There are relationships between the (in)security of a compact riemannian manifold and its topological entropy, fundamental group, flatness of the metric, etc. For instance, the uniform security of $M$ implies the vanishing of its topological entropy and the quasi-nilpotence of $\pi_{1}(M)$ [2]. If, in addition, $M$ has no conjugate points, then it is flat [2]. The current conjecture is that a compact riemannian manifold is uniformly secure iff it is flat [2, 13].

Set $m_{t}(x, y)=\left|\Gamma_{t}(x, y)\right|$ and $n_{t}(x, y)=\left|G_{t}(x, y)\right|$. These are the counting functions for geodesics in $M$. Burns and Gutkin [2] related the security of $M$ with the growth of counting functions as $t \rightarrow \infty$. The goal of the present paper is to establish relationships between the growth of functions $n_{t}(x, y)$ and the asymptotics of $s_{t}(x, y)$. This extends the approach of [2]. To see this, observe that i) a pair $x, y \in M$ is secure iff $s_{t}(x, y)$ is a bounded function on $\mathbb{R}_{+}$; ii) a manifold $M$ is uniformly secure iff the the function $s(t)$ is bounded.

We will relate the topological entropy, volume entropy, and growth rate of $\pi_{1}(M)$ with the asymptotics of functions $s_{t}(x, y)$ and $s(t)$. Before stating our results, we need to say a few words about infinite blocking costs. For almost all $x, y \in M$ we have $n_{t}(x, y)<\infty$ [1, and hence $s_{t}(x, y)<\infty$ as well. Examples show that for some $x, y \in M$ and $t>t_{0}(x, y)$ we may have $m_{t}(x, y)=\infty$ and $s_{t}(x, y)=\infty$, implying $s(t)=\infty$. In view of this, we will often make provisos for the possibility of infinite blocking costs. The following proposition is a combination of Theorem 1 and Theorem 2 in section 3 below .

Theorem. Let $M$ be a compact riemannian manifold.

i) If $\pi_{1}(M)$ grows exponentially, then the blocking cost is either infinite or grows (at least) exponentially.

ii) Let $e>0$ be the topological entropy or the volume entropy of $M$. Then the blocking cost is either infinite or grows exponentially, with the rate greater than or equal to $e / 2$.

The plan of the paper is as follows. In section 1 we expose the background material; we also sketch a proof of Proposition 1 which is 
our key technical result. Our proof explains the relationship between the counting and blocking of geodesics. In section 2 we expose several auxiliary propositions that we will need in section 3 . In section 3 we present our main results.

Notes and acknowledgements. I thank Keith Burns for comments on a draft of the paper.

\section{Background And Preliminaries}

It is convenient to partition the exposition into several subsections.

1.1. Counting geodesics between points, topological entropy, and volume entropy. Let $M$ be a compact riemannian manifold, let $d \mu$ be the riemannian measure, and let $h=h(M)$ be the topological entropy of $M$. Let $\tilde{M}$ be the universal cover of $M$. For $\tilde{x} \in \tilde{M}$ let $B(\widetilde{x}, t)$ be the ball of radius $t$ in $\tilde{M}$ around $\tilde{x}$. The exponential growth rate for $t \mapsto \operatorname{Vol} B(\tilde{x}, t)$ does not depend on $\tilde{x}$; this is the volume entropy $\lambda=\lambda(M)$. The two entropies are related by $\lambda \leq h$, and we have

$$
h=\lim _{t \rightarrow \infty} \frac{1}{t} \log \int_{M \times M} n_{t}(x, y) d \mu(x) d \mu(y) .
$$

If $M$ has no conjugate points, then for any $x, y \in M$ we have

$$
h=\lim _{t \rightarrow \infty} \frac{1}{t} \log n_{t}(x, y) .
$$

See [16] and [15] for this material. Equation (2) fails, in general, if $M$ has conjugate points [3, 4]. From the obvious inequality

$$
s_{t}(x, y) \leq m_{t}(x, y) \leq n_{t}(x, y)
$$

and results of [1], we have $s_{t}(x, y)<\infty$ for almost all (resp. all) $x, y \in M$ (resp. if $M$ has no conjugate points). The manifold is called secure (resp. uniformly secure, resp. totally insecure) if $t \mapsto s_{t}(x, y)$ is bounded for all $x, y \in M$ (resp. $t \mapsto s(\cdot)$ is bounded, resp. $t \mapsto s_{t}(x, y)$ is unbounded for all $x, y \in M)$. The following proposition combines results of [2, 13, 11].

Theorem. Let $M$ be a compact riemannian manifold.

1. If $M$ is uniformly secure, then it has zero topological entropy and virtually nilpotent fundamental group. If, in addition, $M$ has no conjugate points then it is flat.

2. If $M$ has no conjugate points and positive topological entropy, then it is totally insecure.

3. Let $M$ be a locally symmetric space. Then $M$ is secure iff it is uniformly secure iff it is of euclidean type. 
These results suggest the following conjecture [2, 13].

Conjecture 1. A compact riemannian manifold is secure iff it is uniformly secure iff it is flat.

1.2. Estimating the number of geodesics between points via the blocking cost. For the benefit of the reader, we will sketch a proof of Proposition 1 which estimates the counting function for geodesics via the blocking cost.

Let $B(x, y ; t)$ be a minimal blocking set for $\Gamma_{t}(x, y)$. Every $\gamma \in$ $\Gamma_{t}(x, y)$ is a concatenation: $\gamma=\{\alpha \in \Gamma(x, z)\} \cup\{\beta \in \Gamma(z, y)\}$, where $z \in B(x, y ; t)$. Carefully choosing the point $z \in B(x, y ; t)$, we obtain the bound $m_{t}(x, y) \leq \sum_{(p, q) \in \mathcal{P}_{1}} m_{t / 2}(p, q)$; the set $\mathcal{P}_{1} \in M \times M$ consists of pairs $(x, z),(z, y)$, where $z \in B(x, y ; t)$. Hence $\left|\mathcal{P}_{1}\right| \leq 2 s(t)$.

Iterating this argument, we obtain a sequence of finite sets $\mathcal{P}_{k} \in$ $M \times M$ and bounds $m_{t}(x, y) \leq \sum_{(p, q) \in \mathcal{P}_{k}} m_{t / 2^{k}}(p, q)$, where

$$
\left|\mathcal{P}_{k}\right| \leq 2^{k} s(t) \times \cdots \times s\left(\frac{t}{2^{k-1}}\right) .
$$

The inductive process stops when $t / 2^{k}$ gets smaller than the injectivity radius of $M$; we then have $m_{t / 2^{k}}(p, q) \leq 1$ for any pair $p, q \in M$. Let $\kappa(t)$ be the smallest $k \in \mathbb{N}$ satisfying this inequality, and set $S(t)=$ $s(t) \times \cdots \times s\left(\frac{t}{2^{\kappa(t)-1}}\right)$. Then we have the bound

$$
m_{t}(x, y) \leq 2^{\kappa(t)} S(t) .
$$

Since $\kappa(t) \leq$ const $\log _{2} t$, and $n_{t}(x, y) \leq$ const $t^{2} m_{t}(x, y)$, by Lemma 3.1 in [2], we have obtained a desired bound.

1.3. Amplifications. The framework of security makes sense for any space with rich sets of distinguished curves joining arbitrary pairs of points in the space. In particular, it is meaningful for riemannian manifolds with boundaries and corners. Planar billiard tables yield elementary examples of this setting; billiard orbits play the role of riemannian geodesics.

A polygon $P \subset \mathbb{R}^{2}$ is secure if all billiard orbits connecting an arbitrary pair of points in $P$ can be blocked by a finite set. Which polygons are secure and which are insecure? A complete answer is unknown but there are a few partial results [8, 9]. For instance, the regular $n$-gon $R_{n}$ is secure iff $n=3,4,6\left[8\right.$. The counting functions $n_{t}(x, y)$ are subexponential for polygons [10]; it is widely believed that they are, in fact, polynomial [7]. Thus, the results on insecurity of polygons in [8, 9] are obtained using entirely different considerations. 
P. Herreros studied the security for pairs of points in a $C^{1}$ riemannian surface $M$ homeomorphic to the 2-sphere [12]. Herreros found a large set of secure pairs of points whose blocking sets have unexpected properties. This phenomenon has applications to the security in riemannian products $M \times N$.

\section{Blocking COST AND GROWTH OF JOINING GEODESICS}

Let $M$ be a compact riemannian manifold. For $x, y \in M$ and $0<t$ let $s_{t}(x, y)$ be the minimal number of points needed to block all $\gamma \in$ $\Gamma_{t}(x, y)$, and set

$$
s(t)=\sup _{x, y \in M} s_{t}(x, y) \leq \infty .
$$

Then $s_{t}(x, y)$ is the blocking threshold for the triple $(x, y ; t)$, and $s(t)$ is the blocking cost function for $M$. If $s(t)=\infty$ for some $t$, we will say that the blocking cost is infinite 1

We will now introduce an operation on functions in $\mathbb{R}_{+}$; although it is defined in a greater generality, we will restrict our attention to positive, non-decreasing functions. The operation depends on a positive parameter, $\delta$, whose value will be set later on. For the moment, $\delta$ is arbitrary, and we suppress it from notation.

For $t \in \mathbb{R}_{+}$let $\kappa(t) \in \mathbb{N}$ be the smallest $k$ such that $\frac{t}{2^{k}}<\delta$. Equivalently, $\kappa(t)=0$ if $t<\delta$ and $\kappa(t)=1+\left\lfloor\log _{2} t-\log _{2} \delta\right\rfloor$ if $\delta \leq t$. We will denote functions on $\mathbb{R}_{+}$before they are fed into our operation by $f, g$ etc; we use $F, G$ etc for the respective functions produced by the operation.

Let $f$ be a function on $\mathbb{R}_{+}$. We set

$$
F(t)=\prod_{0 \leq k \leq \kappa(t)-1} f\left(\frac{t}{2^{k}}\right) .
$$

Proposition 1. Let $M$ be a compact riemannian manifold with finite blocking cost $s(\cdot)$, and let $\delta$ be its injectivity radius. Let $S(\cdot)$ be the function associated with $s(\cdot)$ by equation (4). Then for any $x, y \in M$ and $0<t$ we have

$$
n_{t}(x, y) \leq \frac{t^{3}}{2 \delta^{3}} S(t)
$$

\footnotetext{
${ }^{1}$ If the blocking threshold for a particular $(x, y ; t)$ is infinite, then the blocking cost is infinite. Although it is apriori possible that $s_{t}(x, y)<\infty$ for all $(x, y ; t)$ but the blocking cost is infinite, no examples are known to the author.
} 
Proof. For $p, q \in M$ and $0<t$ denote by $B_{t}(p, q) \subset M \backslash\{p, q\}$ a minimal blocking set for $\Gamma_{t}(p, q)$.

For $t \in \mathbb{R}_{+}$and any pair $x, y \in M$ we will define a sequence $\mathcal{P}_{k}$ of finite subsets of $M \times M$, where $0 \leq k \leq \kappa(t)$.

If $t<\delta$ then $\kappa(t)=0$ and $\mathcal{P}_{0}=\{(x, y)\}$. This defines the sequence $\mathcal{P}_{k}$ in this case. Let $\delta \leq t$. Set $\mathcal{P}_{1}=\left\{(x, z): z \in B_{t}(x, y)\right\} \cup\{(z, y)$ : $\left.z \in B_{t}(x, y)\right\}$.

Suppose that $\mathcal{P}_{1}, \ldots, \mathcal{P}_{k}$ have been defined. If $\frac{t}{2^{k}}<\delta$ then $k=\kappa(t)$, and we have terminated the sequence of sets. Otherwise we define $\mathcal{P}_{k+1} \subset M \times M$ as the set comprised by pairs $(p, z),(z, q)$ where $(p, q) \in$ $\mathcal{P}_{k}$ and $z \in B_{t / 2^{k}}(p, q)$.

By the argument of Lemma 3.2 in [2], for any $0 \leq k \leq \kappa(t)$ we have

$$
m_{t}(x, y) \leq \sum_{(p, q) \in \mathcal{P}_{k}} m_{t / 2^{k}}(p, q) .
$$

Set $\mathfrak{P}=\mathfrak{P}(x, y ; t)=\mathcal{P}_{\kappa(t)}(x, y)$. Applying equation (5) to $k=\kappa(t)$ and taking into account that $m_{s}(p, q) \leq 1$ if $s<\delta$, we obtain

$$
m_{t}(x, y) \leq|\mathfrak{P}| \text {. }
$$

Minimal blocking sets are not unique, in general. Thus the set $\mathfrak{P} \subset$ $M \times M$ is not uniquely defined, but we will use a bound on $|\mathfrak{P}|$.

We have $\left|\mathcal{P}_{0}\right|=1$; for $1 \leq k \leq \kappa(t)$ we have, by construction

$$
\left|\mathcal{P}_{k}(x, y ; t)\right| \leq 2 s(t) \times 2 s\left(\frac{t}{2}\right) \times \cdots \times 2 s\left(\frac{t}{2^{k-1}}\right)=2^{k} S_{k}(t) .
$$

Since $S_{\kappa(t)}(\cdot)=S(\cdot)$, and $\kappa(t) \leq 1+\log _{2} t-\log _{2} \delta$, equation (7) yields

$$
m_{t}(x, y) \leq \frac{2 t}{\delta} S(t)
$$

By Lemma 3.1 in [2]

$$
m_{t}(x, y) \leq n_{t}(x, y) \leq \frac{t^{2}}{4 \delta^{2}} m_{t}(x, y) .
$$

Combining equations (8), (9), we obtain the claim.

For functions on $\mathbb{R}_{+}$, we will use the standard notation $f=O(g), f=$ $o(g), f \sim g$. The latter means that there are constants $0<c \leq C<\infty$ such that

$$
c f(t) \leq g(t) \leq C f(t) .
$$

We define the rate of exponential growth of a function by

$$
r=r(f)=\limsup _{t \rightarrow \infty} \frac{\log f(t)}{t} .
$$


Equivalently, $r(f)=\inf \left\{a \in \mathbb{R}_{+}: f=O\left(e^{a t}\right)\right\}$. If $0<r<\infty$ (resp. $r=0, r=\infty$ ) then $f$ grows exponentially (resp. subexponentially, super-exponentially).

If $f=O\left(t^{r}\right)$ for some $0 \leq r$, we say that $f$ grows (at most) polynomially. If $f \neq O\left(t^{r}\right)$ for any $r \in \mathbb{R}_{+}$, we say that $f$ is super-polynomial.

Lemma 1. Let $f, g$ be functions on $\mathbb{R}_{+}$, and let $F, G$ be the functions obtained from them via equation (4).

i) If $g=O(f)$ then there exists $\alpha \in \mathbb{R}$ such that $G=O\left(t^{\alpha} F\right)$.

ii) If $g=o(f)$ then $G=O\left(t^{\beta} F\right)$ for any $\beta \in \mathbb{R}$.

iii) If $f \sim g$ then there exist $\alpha, \beta \in \mathbb{R}$ such that $G=O\left(t^{\alpha} F\right), F=$ $O\left(t^{\beta} G\right)$.

Proof. We use equation (44) and the inequality

$$
\log _{2} t-\log _{2} \delta \leq \kappa(t) \leq \log _{2} t-\log _{2} \delta+1 .
$$

Directly from the definition we see that $g=f_{1} f_{2}$ implies $G=F_{1} F_{2}$ and that $f \leq g$ implies $F \leq G$.

Claim i) follows from preceding observations and from the calculation of correspondence $f \rightarrow F$ when $f$ is a constant function. We leave the latter to the reader.

Let now $g=o(f)$. Then for any $0<\varepsilon$ we have the representation $f=\varphi_{\varepsilon} \cdot g$ where $\varphi_{\varepsilon}(t)<\varepsilon$ when $t_{\varepsilon}<t$. Let $0<c$ be the maximum of $\varphi_{\varepsilon}$ on $\left[0, t_{\varepsilon}\right]$. Set $\psi_{\varepsilon}(t)=c$ if $t \leq t_{\varepsilon}$ and $\psi_{\varepsilon}(t)=\varepsilon$ if $t_{\varepsilon}<t$. Then $f \leq \psi_{\varepsilon} \cdot g$. Applying preceding remarks and directly calculating the correspondence $\psi_{\varepsilon} \rightarrow \Psi_{\varepsilon}$, we obtain ii).

Finally, iii) is a direct consequence of i), since $f \sim g$ means that $f=O(g), g=O(f)$.

We will use Proposition 1 to relate the growth of counting functions $n_{t}(x, y)$, as $t \rightarrow \infty$, and the asymptotics at infinity of the blocking cost. We will need a technical lemma.

Lemma 2. Let $f$ be a function on $\mathbb{R}_{+}$, and let $F$ be the function associated with it by equation (4). Then the following statements hold.

i) If $f=O\left(e^{a t}\right)$ then for any $0<\varepsilon$ we have $F=O\left(e^{(2 a+\varepsilon) t}\right)$.

ii) Let $f=O\left(t^{r}\right)$ where $0 \leq r$. Then there exists $\alpha \in \mathbb{R}$ such that $F=O\left(t^{\alpha \log _{2} t}\right)$.

Proof. We have $f(t)<c \cdot e^{a t}$ for some $0<c$. A direct calculation from equation (4) yields $e^{a t} \rightarrow e^{2 a t}$. Computing $F$ when $f=$ const, and using the argument of Lemma 1, we obtain $F=O\left(t^{\alpha} e^{2 a t}\right)$ for some $\alpha \in \mathbb{R}$. This implies i). 
We compute $F$ when $f(t)=t$. Directly from equation (44), we obtain $F(t)=t^{\kappa(t)} 2^{-\kappa(t)(\kappa(t)+1) / 2}$. Estimating $\kappa(t)$ via equation (10), we obtain $F=O\left(t^{a+\frac{1}{2} \log _{2} t}\right)$. Whatever is the value of $a \in \mathbb{R}$, we have $F=$ $O\left(t^{\left(\varepsilon+\frac{1}{2}\right) \log _{2} t}\right)$ for any $0<\varepsilon$.

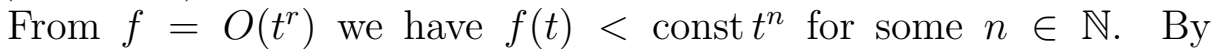
preceding remarks, this implies $F=O\left(t^{a} \cdot t^{n\left(\varepsilon+\frac{1}{2}\right) \log _{2} t}\right)$ for some $a \in \mathbb{R}$. Setting $\alpha=(2 n+1) / 2$, we obtain ii).

Let $f, g$ be positive functions on $\mathbb{R}_{+}$. If $f=O(g)$ (resp. $f=o(g)$ ), we say that $f$ grows not faster (resp. slower) than $g$.

Lemma 3. Let $M$ be a compact riemannian manifold. Suppose that for any $0<c$ there exist $x, y \in M$ such that $n_{t}(x, y)$ grows faster than $t^{c \log t}$. Then the blocking cost is either infinite or super-polynomial.

Proof. Assume the opposite, i. e. that $s=O\left(t^{r}\right)$ for some $0<r$. Then, by Lemma 2, $S=O\left(t^{a \log t}\right)$ for some $0<a$. Proposition 1 yields that $n_{t}(x, y)=O\left(t^{c \log t}\right)$ for any $c$ greater than $a$, and arbitrary $x, y \in M$. This contradicts to the assumption.

\section{BLOCKING THRESHOLDS AND GEOMETRY OF A MANIFOLD}

Let $G$ be a finitely generated group, and let $W(G, S, n) \subset G$ be the set of elements of length at most $n$ with respect to a finite generating set $S$. Denote by $r_{S}(G)$ the growth rate of the function $w_{S}(n)=$ $|W(G, S, n)|$. In general, $r_{S}(G)$ depends on the choice of $S$. We say that $r_{S}(G)$ is the growth rate of $G$ with respect to the set $S$ of generators. Let $S^{\prime}, S^{\prime \prime}$ be two finite sets of generators, and let $r_{S^{\prime}}(G), r_{S^{\prime \prime}}(G)$ be the corresponding rates. Then $0<r_{S^{\prime}}(G)$ iff $0<r_{S^{\prime \prime}}(G)$. Thus, we can speak of groups with exponential growth (resp. groups with subexponential growth) without specifying the growth rate.

The proposition below shows that the blocking cost function controls the topological entropy of a manifold, as well as the growth rate of the fundamental group.

Proposition 2. Let $M$ be a compact riemannian manifold, with a finite blocking $\operatorname{cost} s(\cdot)$.

i) Let $0<\sigma$. If $s=O\left(e^{\sigma t}\right)$ then $h(M) \leq 2 \sigma$.

ii) If the function $s(\cdot)$ grows subexponentially, then the group $\pi_{1}(M)$ grows subexponentially as well.

Proof. i) If $f=O\left(e^{\sigma t}\right)$, then, by Lemma 2, $F=O\left(e^{(2 \sigma+\varepsilon) t}\right)$ for any $0<\varepsilon$. By Lemma 1 and Proposition 1, $n_{t}(x, y)=O\left(e^{(2 \sigma+\varepsilon) t}\right)$ for all $x, y \in M$. By Mañe's formula equation (11), we have $h(M) \leq 2 \sigma+\varepsilon$. Since $\varepsilon$ is arbitrary, we obtain the claim. 
ii) By [17, 18], $\pi_{1}(M)$ grows subexponentially iff $\lambda(M)=0$. By i) and Manning's inequality [16], if $s(\cdot)$ is subexponential then $\lambda(M)=0$.

We will now turn to the main results of this paper.

Theorem 1. Let $M$ be a compact manifold. If the fundamental group of $M$ grows exponentially then the blocking cost for any riemannian metric on $M$ is either infinite or grows at least exponentially.

Proof. If the blocking cost function is subexponential, then, by Proposition 2, $\pi_{1}(M)$ grows subexponentially.

Theorem 2. Let $M$ be a compact riemannian manifold. Let $\lambda=$ $\lambda(M)$ and $h=h(M)$ be its volume entropy and the topological entropy respectively.

i) If $0<h$ then the blocking cost is either infinite or grows exponentially, with the rate at least $h / 2$.

ii) If $0<\lambda$ then the blocking cost is either infinite or grows exponentially, with the rate at least $\lambda / 2$.

Proof. Let $r$ be the exponential growth rate of $s(\cdot)$. By Proposition 2 , $r \geq h / 2$. This proves i). Claim ii) follows from i) by Manning's inequality.

Theorem 3. Let $M$ be a compact riemannian manifold. Suppose that there is $\tilde{x} \in \tilde{M}$ such that $\operatorname{Vol} B(\tilde{x}, t)$ grows faster than any $t^{c \log t}$. Then the blocking cost for $M$ is either infinite or grows super-polynomially.

Proof. Assume the opposite, i. e., that $s=O\left(t^{r}\right)$ for some $0<r$. By Lemma 2 and Proposition 1, there exists $c \in \mathbb{R}_{+}$such that $n_{t}(x, y)=$ $O\left(t^{\log t}\right)$ for all $x, y \in M$. By the proof of Proposition 2, this implies $\operatorname{Vol} B(\tilde{x}, t)=O\left(t^{c \log t}\right)$ for all $\tilde{x} \in \tilde{M}$, contrary to the assumption.

Preceding statements concern the blocking cost function, as opposed to individual blocking thresholds $s_{t}(x, y)$. We will now obtain estimates for those.

Corollary 1. Let $M$ be a compact riemannian manifold. Let $h$ be its topological entropy. If $0<h$, then for any $C>1$, arbitrarily small $\varepsilon>0$, and arbitrarily large $t$ there exist points $x, y \in M$ such that

$$
C e^{\left(\frac{h}{2}-\varepsilon\right) t} \leq s_{t}(x, y) .
$$

Proof. Assume the opposite, i. e. that there is $C>1, \varepsilon>0$ and $\tau \in \mathbb{R}_{+}$ such that for all $x, y \in M$ and all $t$ greater than $\tau$ we have the bound $s_{t}(x, y)<C e^{\left(\frac{h}{2}-\varepsilon\right) t}$. Thus, $s=O\left(e^{\left(\frac{h}{2}-\varepsilon\right) t}\right)$. Hence, the exponential 
growth rate of the blocking cost function is strictly less than $h / 2$. By Theorem 2, this is impossible.

Corollary 2. Let $M$ be a compact riemannian manifold. Let $\lambda$ be its volume entropy. If $0<\lambda$, then for any $C>1$, arbitrarily small $\varepsilon>0$, and arbitrarily large $t$ there exist points $x, y \in M$ such that

$$
C e^{\left(\frac{\lambda}{2}-\varepsilon\right) t} \leq s_{t}(x, y) .
$$

Proof. The claim follows from Corollary 1 and the inequality $\lambda \leq h$ [16].

Corollary 3. Let $M$ be a compact manifold whose fundamental group grows exponentially. We endow $M$ with a riemannian metric. For $x, y \in M$ and $t \in \mathbb{R}_{+}$let $s_{t}(x, y)$ be the blocking threshold with respect to this metric.

Then for any $r>1$ and arbitrarily large $t$ there exist points $x, y \in M$ such that

$$
t^{r}<s_{t}(x, y)
$$

Proof. Suppose that the claim is false. Then there exist $r>1$ and $\tau \in \mathbb{R}_{+}$such that $s_{t}(x, y) \leq t^{r}$ for all $\tau<t$ and $x, y \in M$. Then the blocking cost $s(\cdot)$ is polynomial. This contradicts Theorem 1 .

Remark 1. Corollary 3 says that if the fundamental group of a manifold grows exponentially, then its blocking thresholds are super-polynomial. By our methods we can obtain other statements of that nature. For instance, if $\pi_{1}(M)$ grows faster than any $n^{a \log n}$, then the blocking thresholds for any riemannian metric on $M$ are super-polynomial. This is proved by combining Lemma 2 and Lemma 3 . An analogous statement holds if we replace the growth of $\pi_{1}(M)$ by that of the volume of balls in $\tilde{M}$. We leave details to the reader.

In order to illustrate the preceding material, we will now derive some of the results of [2] and [13].2

Theorem 4. Let $M$ be a uniformly secure compact riemannian manifold. Then the topological entropy for the geodesic flow on $M$ vanishes. The fundamental group of $M$ is virtually nilpotent.

Proof. In the present terminology, the blocking cost function for $M$ is bounded. Hence, by Proposition $2, h(M) \leq 2 a$ for any positive $a$. This proves the first claim.

Let $f$ be a bounded function on $\mathbb{R}_{+}$, and let $F$ be the function corresponding to $f$ via equation (4). As we have seen in the proof of

\footnotetext{
${ }^{2}$ Compare theorem 4 with theorem 4.3 in [2].
} 
Lemma 1, if $f$ is bounded then $F$ grows polynomially. Applying this remark to the blocking cost function of $M$ and using Proposition 1, we obtain a uniform bound $n_{t}(x, y)<$ const $t^{r}$. By the proof of Proposition 2, the group $\pi_{1}(M)$ has polynomial growth. Our second claim now follows from a theorem of Gromov [5].

Remark 2. If the manifold in Theorem 4 has no conjugate points, then the conclusion is much stronger. Namely, by a theorem of Lebedeva, a compact riemannian manifold with no conjugate points and a quasinilpotent fundamental group is flat [14. See theorem 4.3 in [2]. This is one of the results supporting Conjecture 1 .

The following proposition strengthens Theorem 4.5 in [2] 3

Theorem 5. Let $M$ be a compact riemannian manifold with no conjugate points. Let $h>0$ be its topological entropy. Then for any $x, y \in M$ the blocking threshold $s_{t}(x, y)$ grows exponentially. Its exponential growth rate is at least $h / 2$.

Proof. Let $x, y \in M$ be arbitrary, and let $t \in \mathbb{R}_{+}$. The beginning of the proof of Proposition 1 yields

$$
m_{t}(x, y) \leq \sum_{(p, q) \in \mathcal{P}_{1}} m_{t / 2}(p, q)
$$

where $\left|\mathcal{P}_{1}\right|=2 s_{t}(x, y)$. Let $\delta>0$ be the injectivity radius of $M$. By equations (2), (9), we have

$$
\lim _{t \rightarrow \infty} \frac{1}{t} \log m_{t}(p, q)=h .
$$

Let $\varepsilon>0$ be arbitrary. Combining equations (11), (12), we obtain for $t>t(\varepsilon)$,

$$
e^{(h-\varepsilon) t} \leq m_{t}(x, y) \leq 2 s_{t}(x, y) e^{\frac{1}{2}(h+\varepsilon) t} .
$$

Equation (13) yields $e^{\left(\frac{h}{2}-\frac{3 \varepsilon}{2}\right) t} \leq 2 s_{t}(x, y)$; letting $\varepsilon \rightarrow 0$, we obtain the claim.

Applying Theorem 5 to manifolds with nonpositive curvatures, we obtain the following proposition. It strengthens Corollary 4.7 in [2].

Corollary 4. Let $M$ be a compact riemannian manifold of nonpositive curvature. Then the following dichotomy holds:

\footnotetext{
${ }^{3}$ Theorem 4.5 has been independently obtained by Lafont and Schmidt [13].

${ }^{4}$ Here we use that $M$ has no conjugate points. Convergence in equation (12) is uniform [15].
} 
i) The manifold is uniformly secure. Its security threshold is bounded above in terms of the dimension of $M$;

ii) The blocking thresholds $s_{t}(x, y)$ grow exponentially. Their growth rates are greater than or equal to half the topological entropy of $M$.

Proof. By [6, 19], we have the dichotomy: i) $M$ is flat; ii) $M$ has positive topological entropy. In case i), Proposition 2 in [11] yields the claim. In case ii), the claim follows from Theorem 5 .

\section{REFERENCES}

[1] M. Berger and R. Bott, Sur les variétés à courbure strictement positive, Topology 1 (1962), $302-311$.

[2] K. Burns and E. Gutkin, Growth of the number of geodesics between points and insecurity for Riemannian manifolds, preprint math.DS/0701579 (2007).

[3] K. Burns and G. Paternain, On the growth of the number of geodesics joining two points, Pitman Res. Notes Math. 362 (1996), 7 - 20.

[4] K. Burns and G. Paternain, Counting geodesics on a Riemannian manifold and topological entropy of geodesic flows, Erg. Theo. \& Dyn. Sys. 17 (1997), $1043-1059$.

[5] M. Gromov, Groups of polynomial growth and expanding maps, Inst. Hautes Études Sci. Publ. Math. 53 (1981), 53 - 73.

[6] L.W. Goodwyn, Topological entropy bounds measure-theoretic entropy, Proc. AMS 23 (1969), 679 - 688.

[7] E. Gutkin, Billiard dynamics: A survey with the emphasis on open problems, Reg. \& Chaot. Dyn. 8 (2003), 1 - 13.

[8] E. Gutkin, Blocking of billiard orbits and security for polygons and flat surfaces, GAFA: Geom. \& Funct. Anal. 15 (2005), 83 - 105.

[9] E. Gutkin, Insecure configurations in lattice translation surfaces, with applications to polygonal billiards, Discr. Cont. Dyn. Sys. A 16 (2006), 367 - 382.

[10] E. Gutkin and N. Haydn, Topological entropy of polygon exchange transformations and polygonal billiards., Erg. Theo. \& Dyn. Sys. 17 (1997), 849 867.

[11] E. Gutkin and V. Schröder, Connecting geodesics and security of configurations in compact locally symmetric spaces, Geometriae Dedicata 118 (2006), 185 208.

[12] P. Herreros, Blocking: New examples and properties of products, preprint arXiv:0707.0456 (2007).

[13] J.-F. Lafont and B. Schmidt, Blocking light in compact Riemannian manifolds, Geometry \& Topology 11 (2007), 867 - 887.

[14] N.D. Lebedeva, On spaces of polynomial growth with no conjugate points, St. Petersburg Math. J. 16 (2005), 341 - 348.

[15] R. Mañé, On the topological entropy of geodesic flows, J. Diff. Geom. 45 (1997), $74-93$.

[16] A. Manning, Topological entropy for geodesic flows, Ann. Math. 110 (1979), $567-573$.

[17] A. Manning, Relating exponential growth in a manifold and its fundamental group, Proc. AMS 133 (2004), 995 - 997. 
[18] J. Milnor, A note on curvature and fundamental group, J. Diff. Geom. 2 (1968), $1-7$.

[19] Ya. Pesin, Formulas for the entropy of the geodesic flow on a compact Riemannian manifold without conjugate points, Math. Notes 24 (1978), 796 805.

imPA, Estrada Dona Castorina 110, Rio de Janeiro, 22460-320, Brasil; UMK, Chopina 12/18, 87 - 100 Torun, And IMPAN, SNiadeckich 8, 00-956

WarszaWa, POland

E-mail address: gutkin@mat.uni.torun.pl, gutkin@impa.br 